\title{
Biorthogonal Fourier transform for multichirp-rate signal detection over dispersive wireless channel
}

\author{
Lin Zheng ${ }^{1,2^{*}}$ (D), Chao Yang ${ }^{1}$, Chao Yan ${ }^{1}$ and Hongbing Qiu ${ }^{1}$
}

\begin{abstract}
Biorthogonal Fourier transform (BFT), consistent with the matched signal transform (MST), has been introduced to demodulate the M-ray chirp-rate signal which possesses good orthogonality in the BFT domain. Here, we analyze the characteristics of BFT detection in a further step, including the resolution capability of the multichirp-rate signal, the property of pulse compression, the closed-form bit-error rate in the additive white Gaussian noise (AWGN) channel, and the interference in the time-frequency dispersive channel. Even in the high Doppler environment, the shift in BFT detection is proven to be slight. In addition, we deduce that the orthogonality among received chirp rates in the BFT domain would be affected in the multipath dispersive environment. This causes the mutual interference among different chirp rates in a symbol and over symbols concurrently. The theoretical result shows that the chirp modulation parameter can be adjusted to obtain the trade-off between time and frequency dispersion. By the multipath model of chirp-rate signal, an auxiliary parallel interference cancellation (PIC) method is further introduced in multipath environment. Simulations verify our analyzed performance of BFT detection in the AWGN, Doppler, and multipath channels. The proposed interference cancellation algorithms are also proven to be effective.
\end{abstract}

Keywords: Chirp-rate modulation, Biorthogonal Fourier transform, Matched signal transform, Dispersive channel

\section{Introduction}

Early chirp signals, i.e., linear frequency modulation (LFM) signals, are implemented in radar and communication. In radar, the impulse-compression characteristic of chirp is utilized to extract targets, while the chirp signal is invoked as spread-spectrum waveform to suppress interferences in communications [1]. In recent years, wireless sensor networks, as well as military ad hoc network, have the requirement of integration with self localization, sensing, and communication functions. In this context, chirp scheme for localization and communication is unanimously adopted by the IEEE 802.15.4a Working Group in 2005 as a supplemental physical layer standard of wireless sensor network [2]. Meanwhile, many efforts in signal

*Correspondence: gwzheng@gmail.com

${ }^{1}$ Key Laboratory of Guangxi Broadband Wireless Communication \& Signal Processing, Guilin University of Electronic Technology, Jinji Road, 541004 Guilin, China

${ }^{2}$ Key Laboratory of Science and Technology on Communication Networks, Zhongshan Western street, 050081 Shijiazhuang, China processing devote to estimate range or localization with a chirp signal $[3,4]$.

In other fields, broadband wireless communications in high-speed vehicles, such as aircraft and high-speed trains, are much in demand. Conventional phase-based transceivers are unable to meet the needs of the large Doppler shift caused by moving speeds over $200 \mathrm{~km} / \mathrm{h}$ unless they adopt complicated frequency-shift estimation and compensation. The chirp signal is a good candidate since it is a constant-modulus time-frequency signal and does not require phase detection. However, chirp suffers low-modulation efficiency owing to its simplex spreadspectrum waveform.

Some efforts on improving the modulation efficiency of a chirp signal have been reported in recent years. Wysocki proposes a Walsh-coded chirp modulation in [5] for multiple access. Segments with different phases in a symbol are designed to maintain orthogonality among multiuser signals. A multidimensional chirp modulation scheme with code division spread spectrum is presented in [6]. By overlapping subbands and modulating with 
pseudorandom sequences, the scheme improves modulation efficiency in frequency and time domain. Analog and digital correlators are both adopted in [7]. The correlation by the former reduces the time-bandwidth product of a chirp signal and thereby reducing the complexity of post-stage digital processing. To improve orthogonality, [8] introduces a two-segment structured symbol modulated by different chirp rates and Walsh codes. Submitting to the IEEE802.15.4a physical layer proposal, Nanotron Inc. develops a $M$-ary modulation by four-segments linear chirps [9] and employs the multi-choice precoding (MCP) technology to solve the multipath fading problem in noncoherent detection. Its modulation efficiency still needs to be improved, and MCP has poor performance in a fast fading channel due to the requirement of the channel information feedback from receiver to transmitter.

The progress in time-frequency (T-F) signal processing is also applied to chirp detection. Recently, the T-F dispersive channel and orthogonal frequency-division multiplexing (OFDM) signal have been intensively studied [10]. The series of the filter bank multicarrier (FBMC) and T-F filters for OQAM have been proposed to offer better performance to Doppler shift [11]. Here, we concern the various TF-based detectors developed for detecting chirp signals. They include the dechirping technique, the Radon-Wigner transform [12], the Radon-ambiguity transform [13], the chirplet transform [14], and the short-time Fourier transform [15]. A multi-carrier chirp communication system based on the fractional Fourier transform (FRFT) is developed in [16] and has been proven to be more reliable compared to fast Fourier transform (FFT) based OFDM in time-frequency-selective channels. In recent years, FRFT-OFDM has received attention, and its performance has been analyzed in the dispersive environment, especially in the frequency offset or Doppler frequency shift analysis [17-19]. All of them reveal that FRFT detection performance is significantly better. Furthermore, the affine fourier transform as a generalized FRFT is introduced to multicarrier system in [20, 21]. In [22], the out-of-band power reduction methods are proposed to the weighted-type FRFT-based multicarrier system. The detectors mentioned above show better anti-ICI performance, that is proved to be feasible in ground-to-air channels. However, the time dispersion (ISI) evaluation and performance analysis for the chirp multicarrier method is neglected.

Other analyses in [23] and [24] concentrate on the resolution of multi-components in the short-term FRFT and FRFT domain. The condition is deduced to obtain the maximal resolution performance. Tao [31] and Zhao [26] introduce the FRFT to estimate the multiple components of a chirp signal. However, simultaneous FRFT detectors with different orders should be applied to corresponding chirp-rate signals. This, however, results in high computing load and complexity in practice.

Matched signal transforms (MSTs) to exponential instantaneous frequency structures are proposed in [27], and further, a linear MST method is introduced to suppress LFM interference [28]. Wang [29, 30] presents a transform being consistent with linear MSTs, naming it the biorthogonal Fourier transform (BFT), to detect a chirp signal since it matches the chirp rate. Like the Fourier transform to a single-frequency waveform, the impulse-compression effect of the chirp rate is achieved in the BFT domain. Unlike FRFT, the demodulation of the multichirp-rate signal requires only one BFT process to complete. So far, the demodulation performance of BFT or linear MST to chirp-rate signal, and the applicability of BFT in wireless doubly dispersive channels have not yet been analyzed in the literatures.

This paper deduces the output signal-to-noise-ratio (SNR) of BFT detection of chirp-rate signal, and the closed-form bit-error-rate (BER) result is obtained. Some characteristics of the discrete BFT are analyzed to design the multichirp-rate signal and the demodulation algorithm. In a practical environment, we demonstrate that the orthogonality among the chirp-rate signals in the BFT domain will be unfortunately affected by multipath propagation. However, by adjusting the chirp rate of the signaling scheme, we obtain a trade-off between the tolerance of the time and frequency dispersion, i.e., ICI and ISI. In addition, we also developed an auxiliary parallel interference cancellation (PIC) method based on the dispersive model in BFT domain to mitigate the multipath interference. At the end of paper, the effect of the Doppler shift is analyzed and verified by simulations. With square-law $\mathrm{BFT}\left(\mathrm{BFT}^{2}\right)$, outstanding detection performance exceeds that of FRFT and FSK in the channels with large Doppler shift.

The rest of this paper is organized as follows: firstly, multichirp-rate signal models in the time domain is given in Section 2. Secondly, Section 3 gives the definition of BFT and analyzes the BFT characteristics, including the closed-form solution of BFT detection performance. Thirdly, BFT detection in frequency-offset and multipath environments are discussed in Section 4 and Section 5, respectively. Fourthly, based on the multipath model in BFT domain derived in Section 5, a MMSE detection aided by the decision-directed PIC is proposed in Section 6. Fifthly, the simulation results and some discussions are given in Section 7. Finally, the conclusions of our work are summarized in last section.

\section{Multichirp-rate signal model}

A multichirp-rate scheme is introduced to improve the modulation efficiency of a chirp spread-spectrum (CSS) signal $[29,31]$. It maintains the frequency characteristics 
of the original LFM and avoids high demand of the phasemodulated signal for strict synchronization and equalization of the receiver.

At the $n$th symbol, the $M$-ary chirp-rate signal model can be presented as

$$
\begin{aligned}
s_{M}(n, t)= & \sum_{m=1}^{M} b[n M+m] \exp \left(j 2 \pi \left(f_{0}\left(t-n T_{s}\right)\right.\right. \\
& \left.\left.+\frac{1}{2} K_{m}\left(t-n T_{s}\right)^{2}\right)\right),\left((n-1) T_{s} \leq t<n T_{s}\right)
\end{aligned}
$$

where $M$ represents the number of chirp rates, i.e., the $M$-ary modulated symbol. $K_{m}$ is the chirp rate, and $f_{0}$ is the center frequency. $T_{s}$ is the modulated symbol period. $b[k] \in\{-1,1\}$ is the binary information bit. This multiple chirp-rate modulation can be viewed as a parallel $M$-waymodulated signal combination in the chirp-rate domain. Different chirp rate represents different information bit in a symbol period, as shown in Fig. 1.

\section{BFT on chirp-rate signal}

The biorthogonal Fourier transform (BFT) and its inverse conversion algorithm is defined in $[29,30]$ (or refer to [28]):

$$
\begin{aligned}
& \operatorname{BFT}[f(t)] \triangleq F(\beta)=2 \int_{0}^{+\infty} f(t) t \exp \left(-j \beta t^{2}\right) d t \\
& \operatorname{IBFT}[F(\beta)] \triangleq f(t)=\frac{1}{2 \pi} \int_{-\infty}^{+\infty} F(\beta) \exp \left(j \beta t^{2}\right) d \beta
\end{aligned}
$$

Obviously, to the signal of linear frequency modulation $f(t)=\exp \left(j \pi K_{m} t^{2}\right)$, it has BFT $[f(t)]=2 \pi \delta\left(\beta-\pi K_{m}\right)$, where the impulse position at $\pi K_{m}$ in the BFT domain reflects the chirp rate. Referring to the short-term discrete Fourier transform (STDFT), the discrete BFT (D-BFT) performs a circular convolution operation. Here, the oversampling frequency is denoted as $f_{c}$ and its interval is $T_{c}$.
The time and BFT domain are discretized as $t=l T_{c}$ and $\beta=k \Delta \beta$, where $l$ and $k$ are integers. Furthermore, the $N$-point symbol duration is defined as $T_{s}=N T_{c}$ with $N \gg 1$, and $N$-point chirp-rate duration is defined as $\Omega=N \Delta \beta$. Thus, it has the following relationship:

$$
\begin{aligned}
T_{c} & =1 / f_{c}, \Delta \beta=2 \pi / T_{s}^{2} \\
\Omega & =2 \pi f_{c} / T_{s}, \beta t^{2}=k l^{2} \frac{2 \pi}{N^{2}} \\
F(k \Delta \beta) & =\frac{2}{f_{c}} \sum_{l=0}^{N} f\left(l T_{c}\right) l T_{c} \exp \left(-j \frac{2 \pi}{N^{2}} k l^{2}\right)
\end{aligned}
$$

The D-BFT and its inverse transform (D-IBFT) can be derived as

$$
\begin{gathered}
F(k)=\frac{2}{f_{c}} \sum_{l=0}^{N} f(l) l \exp \left(-j \frac{2 \pi}{N^{2}} k l^{2}\right) \\
f(l)=\frac{1}{N^{2}} \sum_{k=0}^{N} F(k) \exp \left(j \frac{2 \pi}{N^{2}} k l^{2}\right)
\end{gathered}
$$

The BFT is shown to be equivalent to the Fourier transform of a nonlinearly sampled or warped version of the signal. Therefore, the FFT can be used to improve its computing efficiency. It can be accomplished by firstly oversampling $f(t)$ to reduce approximation errors in obtaining warped signal samples.

By analyzing the BFT and D-BFT, we derive some characteristics and performances of the transform in multichirp-rate signal detection.

Theorem 1 The BFT impulse position is determined by the time-bandwidth product of chirp-rate signal. When the time-bandwidth product is an integer multiple of 2, the BFT impulse peak is at the discrete $k$.

Proof Suppose chirp-rate signal is $f(t)=$ $A \exp \left(j \pi K_{m} t^{2}\right)$. Its discrete form is $f\left(l T_{c}\right)=$

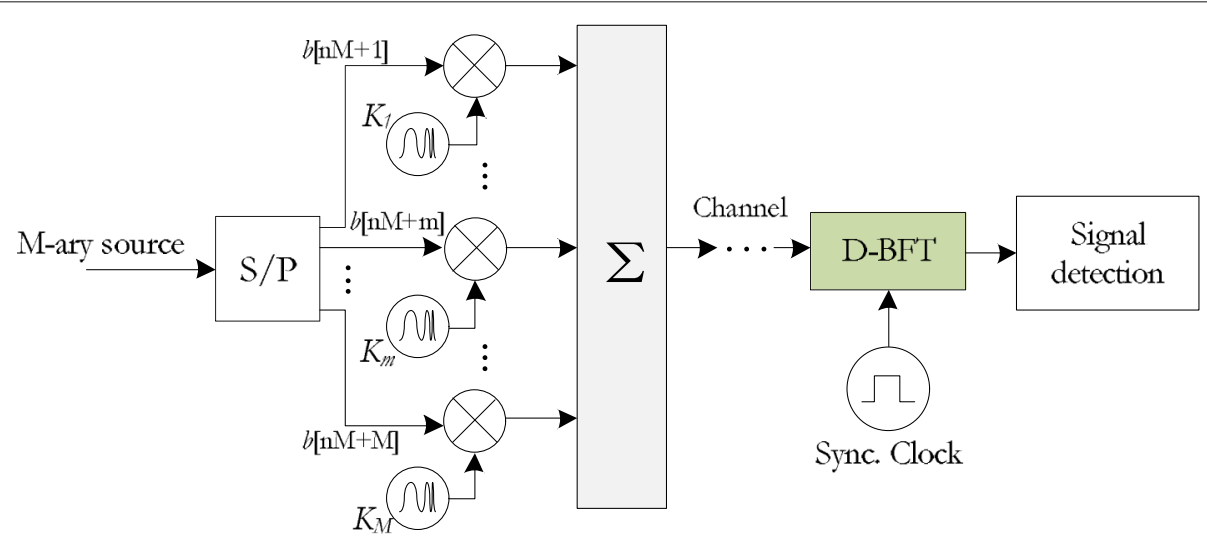

Fig. $1 \mathrm{M}$-ary chirp-rate signal and D-BFT demodulation. The figure shows the system block diagram for the multichirp-rate modulation and BFT demodulation 
$A \exp \left(j \pi K_{m} l^{2} T_{c}^{2}\right)$. Inserting it into the above D-BFT expression, we obtain the peak position $\kappa$ of $F(k)$, which is given by

$$
\kappa=\frac{1}{2} K_{m} T_{c}^{2} N^{2}=\frac{1}{2} K_{m} T_{s}^{2}
$$

According to $K_{m}= \pm B_{m} / T_{s}$ where $B_{m}$ is the bandwidth of chirp-rate signal, we have $\kappa= \pm B_{m} T_{s} / 2$. Therefore, the BFT peak is at the integer $k$ when the $\operatorname{chirp} B_{m} T_{s}$ is a multiple of 2 .

By calculating the BFT at $\kappa= \pm B_{m} T_{s} / 2(m=$ $1,2, \cdots, M)$, the BFT peak of the $m$ th chirp-rate signal is obtained. This operation undoubtedly simplifies the BFT of chirp-rate signal and reduces computational load.

Lemma 1 When the chirp-rate signals have different integer $B_{m} T_{s} / 2$, they are orthogonal to each other at the discrete $k$ in BFT domain.

Proof In a symbol duration $T_{s}$, the BFT of $f(t)=$ $A \exp \left(j \pi K_{m} t^{2}\right),\left(0 \leq t<T_{s}\right)$ is given by

$$
\begin{aligned}
\operatorname{BFT}[f(t)] & =2 A \int_{0}^{T_{s}} t \exp \left(-j\left(\beta-\pi K_{m}\right) t^{2}\right) d t \\
& =A T_{s}^{2} \operatorname{Sa}\left(\frac{\left(\beta-\pi K_{m}\right) T_{s}^{2}}{2}\right) \exp \left(-j\left(\beta-\pi K_{m}\right) T_{s}^{2} / 2\right)
\end{aligned}
$$

which is a $S a(\cdot)$ impulse. Thus, there is

$$
|\operatorname{BFT}[f(t)]|=A T_{s}^{2} \mathrm{Sa}\left(\frac{\left(\beta-\pi K_{m}\right) T_{s}^{2}}{2}\right)
$$

With $\Delta \beta=2 \pi / T_{s}^{2}$, substituting $\beta=\pi K_{m} \pm 2 \pi k / T_{s}^{2}(k \neq$ $0, k= \pm 1, \pm 2, \cdots)$ into the above BFT result, it has

$$
|\operatorname{BFT}[f(t)]|=A T_{s}^{2} \operatorname{Sa}\left(\frac{2 k \pi}{2}\right)=0
$$

In addition, it has the peak $|\operatorname{BFT}[f(t)]|=A T_{s}^{2}$ at $\beta=$ $\pi K_{m}$, that is $k=\beta / \Delta \beta=B_{m} T_{s} / 2$ in the discrete BFT. Therefore, the chirp-rate signals have the orthogonality in discrete BFT domain if they have different integer $B_{m} T_{s} / 2$.

According to the lemma, the multichirp-rate symbol can be demodulated, and the BFT impulses corresponding to different chirp rates can be resolved.
Theorem 2 In the AWGN channel, SNR of the BFT detection to chirp-rate signal depends on the timebandwidth product of the received signal where the bandwidth is the sampling bandwidth. The BER of BFT demodulation is

$$
P_{e}=Q\left(\sqrt{\frac{3}{2} \cdot \frac{E_{b}}{N_{0}}}\right)
$$

where $E_{b}$ is the received chirp-rate energy in a symbol period, and $N_{0}$ is the single-sided power spectral density of AWGN.

Proof In the continuous time domain, the peak amplitude of BFT output is $A T_{s}^{2}$ where $\mathrm{A}$ is the amplitude of the received signal. Owing to the I-Q complex modulation in Eq. (1), the peak power by BFT demodulation is given by

$$
S_{\text {out }}=A^{2} T_{s}^{4}
$$

In the complex zero-mean AWGN environment, the noise can be decomposed into $n(t)=a_{n}(t) \exp (j \phi(t))$, where $a_{n}(t)$ is the amplitude with Rayleigh distribution, and $\phi(t) \in(0,2 \pi]$ is the phase with uniform distribution, and they are statistically independent of each other [32]. Thus, the noise by BFT can be expressed as

$$
\begin{aligned}
N_{\text {out }}(\beta) & =2 \int_{0}^{T_{s}} n(t) t \exp \left(-j \beta t^{2}\right) d t \\
& =2 \int_{0}^{T_{s}} t a_{n}(t) \exp \left(j \phi(t)-j \beta t^{2}\right) d t \\
& =2 \int_{0}^{T_{s}} \operatorname{tn}^{\prime}(t) d t
\end{aligned}
$$

Since $\phi(t)$ follows uniform distribution, $\left(\phi(t)-\beta t^{2}\right)$ also follows uniform distribution. Thus, $n^{\prime}(t)=$ $a_{n}(t) \exp \left(j \phi(t)-j \beta t^{2}\right)$ is still a Gaussian noise due to the independence of $a_{n}(t)$ and $\left(\phi(t)-\beta t^{2}\right)$, which obey Rayleigh distribution and uniform distribution, respectively. Then, the noise variance of the BFT can be represented as

$$
\begin{aligned}
\operatorname{Var}\left[N_{\text {out }}(\beta)\right]= & 4 \iint_{0}^{T_{s}} E\left(t_{1} n^{\prime}\left(t_{1}\right) t_{2} n^{\prime}\left(t_{2}\right)\right) d t_{1} d t_{2} \\
= & 4 \iint_{0}^{T_{s}} t_{1} t_{2} R_{n^{\prime}}\left(t_{1}, t_{2}\right) d t_{1} d t_{2} \\
= & 4 \int_{-T_{s}}^{0} R_{n^{\prime}}(\tau) d \tau \int_{-\tau}^{T_{s}} t_{2}\left(\tau+t_{2}\right) d t_{2} \\
& +4 \int_{0}^{T_{s}} R_{n^{\prime}}(\tau) d \tau \int_{0}^{T_{s}-\tau} t_{2}\left(\tau+t_{2}\right) d t_{2} \\
= & 4 \int_{-T_{s}}^{0} R_{n^{\prime}}(\tau)\left(\frac{1}{2} T_{s}^{2} \tau+\frac{1}{3} T_{s}^{3}-\frac{1}{6} \tau^{3}\right) d \tau \\
& +4 \int_{0}^{T_{s}} R_{n^{\prime}}(\tau)\left(\frac{1}{2}\left(T_{s}-\tau\right)^{2} \tau+\frac{1}{3}\left(T_{s}-\tau\right)^{3}\right) d \tau
\end{aligned}
$$


By the front band-pass filter and sampler in the receiver, $n^{\prime}(t)$ is the Gaussian noise with bandwidth $B=f_{c}$. Therefore, its autocorrelation function is $R_{n^{\prime}}=\sigma_{n}^{2} S_{a}\left(\pi \tau / T_{c}\right)$. We note that $R_{n^{\prime}}(\tau) \approx 0$ when $\tau>T_{c}$. In addition, when $\tau \leq T_{c}$, i.e., $\tau \ll T_{s}$, the item $\frac{1}{6} \tau^{3}$ is negligible with the oversampling times $N \gg 1$ and $\left(T_{s}-\tau\right)^{2} \approx T_{s}^{2}$, $\left(T_{s}-\tau\right)^{3} \approx T_{s}^{3}$. Thus, we have

$$
\begin{aligned}
\operatorname{Var}\left[N_{\text {out }}(\beta)\right] & \approx 4 \int_{-T_{s}}^{T_{s}} R_{n^{\prime}}(\tau)\left(\frac{1}{2} T_{s}^{2} \tau+\frac{1}{3} T_{s}^{3}\right) d \tau \\
& =\frac{4}{3} \sigma_{n}^{2} T_{s}^{3} \int_{-T_{s}}^{T_{s}} S_{a}\left(\frac{\pi \tau}{T_{c}}\right) d \tau=\frac{4}{3} \sigma_{n}^{2} T_{s}^{3} T_{c}
\end{aligned}
$$

The item $R_{n^{\prime}}(\tau) \cdot T_{s}^{2} \tau$ is an odd function of $\tau$, so that its integral over $\left[-T_{s}, T_{s}\right]$ is zero.

We note that the BFT in Eq. (10) is a linear transform, so that $N_{\text {out }}(\beta)$ is still a Gaussian noise. Therefore, the BER of BFT demodulation for the bipolar chirp-rate signal could be derived as

$$
\begin{aligned}
P_{e} & =Q\left(\sqrt{\frac{2 S_{\text {out }}}{\operatorname{Var}\left[N_{\text {out }}(\beta)\right]}}\right) \\
& =Q\left(\sqrt{\frac{3}{2} \cdot \frac{A^{2} T_{s}}{\sigma_{n}^{2} T_{c}}}\right)
\end{aligned}
$$

Without regarding to the processing gain $g=T_{s} / T_{c}=N$, the BER is expressed by $E_{b} / N_{0}=A^{2} T_{s} / \sigma_{n}^{2} T_{c}$ as

$$
P_{e}=Q\left(\sqrt{\frac{3}{2} \cdot \frac{E_{b}}{N_{0}}}\right)
$$

Here, we further deduce the output SNR of the D-BFT. The discretized chirp-rate signal $f\left(l T_{c}\right)=$ $A \exp \left(j \pi K_{m} l^{2} T_{c}^{2}\right)$ is transformed as

$$
\begin{aligned}
S(k) & =\mathrm{D}-\mathrm{BFT}[f(t)] \\
& =\frac{2 A T_{s}}{N} \sum_{l=0}^{N} l \exp \left(j \pi K_{m} l^{2} T_{c}^{2}\right) \exp \left(-j \frac{2 \pi}{N^{2}} k l^{2}\right)
\end{aligned}
$$

At $k^{\prime}=B_{m} T_{s} / 2$, the amplitude of matching impulse by D-BFT is derived to be $S\left(k^{\prime}\right)=(N+1) A T_{s}$.

By expressing the discrete zero-mean AWGN as $n(l)=$ $A_{l} \exp \left(j X_{l}\right)$, where $A_{l}$ meets Rayleigh distribution and $X_{l}$ meets uniform distribution in $(0,2 \pi]$. Similar to the deduction in Eq. (11), substituting $n(l)$ into the D-BFT expression, we have

$$
\begin{aligned}
N(k) & =\mathrm{D}-\mathrm{BFT}[n(t)] \\
& =\frac{2 T_{s}}{N} \sum_{l=0}^{N} l \cdot A_{l} \exp \left(j X_{l}-j \frac{2 \pi}{N^{2}} k l^{2}\right) \\
& =\frac{2 T_{s}}{N} \sum_{l=0}^{N} l \cdot n^{\prime}(k, l)
\end{aligned}
$$

Shown in the expression, the output noise from the D-BFT is a linear accumulation of Gaussian processes. Obviously, it is still a zero-mean Gaussian noise, so that its variance is derived as

$$
\begin{aligned}
\operatorname{Var}[N(k)] & =E\left[\left(\frac{2 T_{s}}{N} \sum_{l=0}^{N} l \cdot n^{\prime}(k, l)\right)^{2}\right] \\
& =\frac{4 T_{s}^{2}}{N^{2}} \sigma_{n}^{2} \sum_{l=0}^{N} l^{2} \\
& =\frac{2 T_{s}^{2}(N+1)(2 N+1)}{3 N} \sigma_{n}^{2}
\end{aligned}
$$

Here, we have the output SNR of the D-BFT on chirprate signal when $N \gg 1$

$$
\frac{S\left(k^{\prime}\right)^{2}}{\operatorname{Var}[N(k)]}=\frac{3 N(N+1)}{2(2 N+1)} \cdot \frac{A^{2}}{\sigma_{n}^{2}} \approx \frac{3 N}{4} \cdot \frac{A^{2}}{\sigma_{n}^{2}}
$$

This result is consistent with the SNR of the BFT to continuous signal, and the additional $N$ in the numerator is the processing gain of the D-BFT demodulator.

\section{Frequency offset in BFT detection}

At the receiver, there is a frequency offset between the received signal and local carrier. This offset is caused either by the difference oscillators in the transmitter and receiver, or by the Doppler shift. The detection performance may be severely degraded as the frequency offset is large and volatile, especially in an OFDM system. Not surprisingly, BFT detection of the chirp-rate signal is affected by frequency offset. The compressed impulse in the BFT domain is shifted and attenuated.

Supposing there is a frequency offset $f_{d}$, the chirp-rate signal is given by

$$
\begin{array}{r}
f_{i}(t)=A \exp \left(j 2 \pi f_{d} t+j \pi K_{m} t^{2}+j \phi_{i}\right), \\
t \in\left(0, T_{s}\right]
\end{array}
$$

where $\phi_{i}$ is the initial random phase of the $i$ th symbol. By BFT, we have

$$
\begin{aligned}
& \operatorname{BFT}\left[f_{i}(t)\right] \\
& =A \int_{0}^{T_{s}} \exp \left(j 2 \pi f_{d} t+j \phi_{i}\right) \exp \left[-j\left(\beta-\pi K_{m}\right) t^{2}\right] d t^{2} \\
& =A \int_{0}^{T_{s}^{2}} \exp \left(j 2 \pi f_{d} \sqrt{V}+j \phi_{i}\right) \exp \left(-j\left(\beta-\pi K_{m}\right) V\right) d V
\end{aligned}
$$


At $\beta=\pi K_{m}$, the discrete sample for the chirp rate in the BFT domain is deduced as

$$
\begin{aligned}
F_{f o}(\beta & \left.=\pi K_{m}\right)=2 A \exp \left(j \phi_{i}(t)\right) \int_{0}^{T_{s}} t \exp \left(j 2 \pi f_{d} t\right) d t \\
& =\frac{A \exp \left(j \phi_{i}(t)\right)\left[\left(1-j 2 \pi f_{d} T_{s}\right) \exp \left(j 2 \pi f_{d} T_{s}\right)-1\right]}{2 \pi^{2} f_{d}^{2}}
\end{aligned}
$$

This is the result of D-BFT detection with a frequencyoffset signal, thereby developing the attenuation expression $\left|F_{f_{o}}\left(\beta=\pi K_{m}\right)\right| / A T_{s}^{2}$.

By Taylor series expansion, $\sqrt{V}$ is extracted as

$$
\sqrt{V}=\sqrt{V_{0}}+\frac{1}{2 \sqrt{V_{0}}}\left(V-V_{0}\right)+\frac{1}{2} \frac{-1}{4 V_{0} \sqrt{V_{0}}}\left(V-V_{0}\right)^{2}+\cdots
$$

According to the range of $V$ in $\left[0, T_{s}^{2}\right]$ and the integral in Eq. (20), the approaching point is near $V_{0}=T_{s}^{2}$. The first two items in the series are left to approximate $\sqrt{V}$; then, we have $\sqrt{V} \approx T_{s} / 2+V /\left(2 T_{s}\right)$. Substituting it into Eq. (20), we obtain

$$
\begin{gathered}
F_{f_{0}}(\beta) \approx A \exp \left(j \phi_{i}\right) \int_{0}^{T_{s}^{2}} \exp \left[j 2 \pi f_{d}\left(\frac{T_{s}}{2}+\frac{V}{2 T_{s}}\right)\right] \\
\cdot \exp \left[-j\left(\beta-\pi K_{m}\right) V\right] d V \\
=A T_{s}^{2} \mathrm{Sa}\left[\left(\beta-\pi K_{m}-\frac{\pi f_{d}}{T_{s}}\right) T_{s}^{2} / 2\right] \\
\cdot \exp \left[-j\left(\beta-\pi K_{m}-\frac{3 \pi f_{d}}{T_{s}}\right) T_{s}^{2} / 2+j \phi_{i}\right]
\end{gathered}
$$

As a result, the compressed impulse in the BFT domain is shifted by about $\pi f_{d} / T_{s}$ from its original $\pi K_{m}$ value. Compared to the interval $\Delta \beta=2 \pi / T_{s}^{2}$ in the D-BFT domain, this shift is small with $f_{d} \ll 1 / T_{s}$ in general.

\section{BFT on multipath signal}

\subsection{Signal analysis in the BFT domain}

In practice, the detector has to be confronted with wireless propagation environment. Here, we analyze the transform on the multipath chirp-rate signal. Without loss of generality, the model of the multipath channel is given by

$$
h(t)=\alpha_{0} \delta(t)+\sum_{l=1}^{L-1} \alpha_{l} e^{j \phi_{l}} \delta\left(t-\tau_{l}\right)
$$

where $L$ is the number of paths and $\tau_{l}$ is the delay of the $l$ th path. A multipath chirp-rate signal can then be expressed as

$$
r(t)=\alpha_{0} \exp \left(j \pi K_{m} t^{2}\right)+\sum_{l=1}^{L-1} \alpha_{l} e^{j \phi_{l}} \exp \left(j \pi K_{m}\left(t-\tau_{l}\right)^{2}\right)
$$

We extract a path component of this signal and analyze it in the BFT domain. By the transform in $t \in\left[0, T_{S}\right]$, we obtain

$$
\begin{aligned}
& \operatorname{BFT}\left[r_{l}(t)\right]= F(\beta) \\
&= \alpha_{l} e^{j \phi_{l}} \int_{\tau_{l}}^{T_{s}} \exp \left(j \pi K_{m}\left(t-\tau_{l}\right)^{2}\right) 2 t \exp \left(-j \beta t^{2}\right) d t \\
&= \alpha_{l} e^{j \phi_{l}} \exp \left(j \pi K_{m} \tau_{l}^{2}\right) \int_{\tau_{l}^{2}}^{T_{s}^{2}} \exp \left(-j 2 \pi K_{m} \tau_{l} \sqrt{U}\right) \\
& \cdot \exp \left(-j\left(\beta-\pi K_{m}\right) U\right) d U, \quad\left(U=t^{2}\right)
\end{aligned}
$$

Similar to the deduction in Eq. (23), the integral above is simplified by Taylor series expansion. $\sqrt{U} \approx T_{s} / 2+$ $U /\left(2 T_{s}\right)$ is substituted in, and further derivation is given by

$$
\begin{gathered}
\mathrm{BFT}\left[r_{l}(t)\right] \approx \\
\alpha_{l} e^{j \phi_{l}} e^{j \pi K_{m} \tau_{l}^{2}} \int_{\tau_{l}^{2}}^{T_{s}^{2}} \exp \left(-j 2 \pi K_{m} \tau_{l}\left(T_{s} / 2\right.\right. \\
\left.\left.+U / 2 T_{s}\right)\right) \exp \left(-j\left(\beta-\pi K_{m}\right) U\right) d U \\
=\alpha_{l} e^{j \phi_{l}} e^{j \pi K_{m}\left(\tau_{l}^{2}-\tau_{l} T_{s}\right)} \int_{\tau_{l}^{2}}^{T_{s}^{2}} \exp \left\{-j\left(\beta-\pi K_{m}\right.\right. \\
\left.\left.+\pi K_{m} \tau_{l} / T_{s}\right) U\right\} d U \\
=\alpha_{l}^{\prime} \operatorname{Sa}\left[\frac{T_{s}^{2}-\tau_{l}^{2}}{2}\left(\beta-\pi K_{m}+\pi K_{m} \tau_{l} / T_{s}\right)\right] e^{j \Phi_{l}(\beta)}
\end{gathered}
$$

where

$$
\begin{aligned}
& \alpha_{l}^{\prime}=\alpha_{l}\left(T_{s}^{2}-\tau_{l}^{2}\right) \text { and } \\
& \begin{array}{r}
\Phi_{l}(\beta)=-\left(\beta-\pi K_{m}+\pi K_{m} \tau_{l} / T_{s}\right) \cdot\left(T_{s}^{2}+\tau_{l}^{2}\right) / 2 \\
\quad+\pi K_{m}\left(\tau_{l}^{2}-\tau_{l} T_{s}\right)+\phi_{l}
\end{array}
\end{aligned}
$$

From the result, it is notable that the impulse in the BFT domain is shifted from $\pi K_{m}$ to $\pi K_{m}\left(1-\tau_{l} / T_{s}\right)$. At the same time, the width of the impulse is also spread from $2 \pi / T_{s}^{2}$ to $2 \pi /\left(T_{s}^{2}-\tau_{l}^{2}\right)$. The longer the delay, the looser the impulse. These results also present the offset and extension of the $\beta$-domain impulse when there exists symbol synchronization error.

On the other hand, the BFT of the multipath signal with cross delay $\tau_{l}$ from the previous symbol is derived in the same way as

$$
\begin{aligned}
& \operatorname{BFT}\left[r_{l}^{-}(t)\right] \approx \\
& \alpha_{l}^{\prime \prime} \operatorname{Sa}\left[\frac{\tau_{l}^{2}}{2}\left(\beta-\pi K_{m}-\pi K_{m}\left(1-\tau_{l} / T_{s}\right)\right)\right] e^{j \Psi_{l}(\beta)}
\end{aligned}
$$

where 


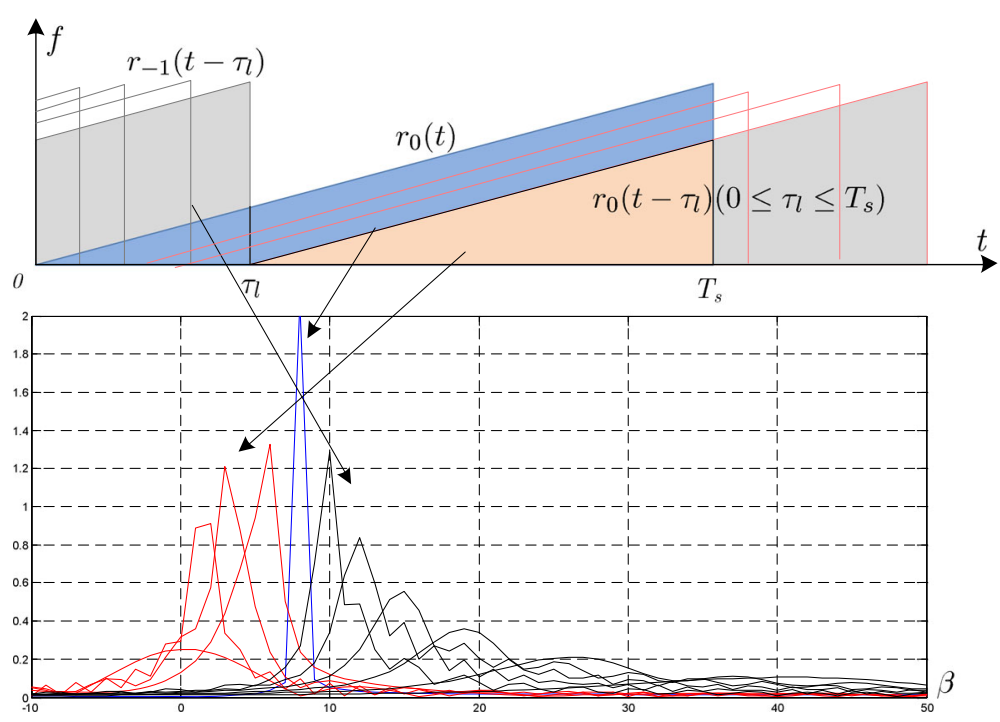

Fig. 2 BFT on the multipath chirp-rate signal. The figure shows the BFT output of the chirp-rate signal in multipath environment

$$
\begin{aligned}
\alpha_{l}^{\prime \prime}= & \alpha_{l} \tau_{l}^{2} \text { and } \\
\Psi_{l}(\beta)= & -\left(\beta-\pi K_{m}-\pi K_{m}\left(1-\tau_{l} / T_{s}\right)\right) \cdot \tau_{l}^{2} / 2 \\
& +\pi K_{m}\left(2 T_{s}^{2}+\tau_{l}^{2}-3 \tau_{l} T_{s}\right)+\phi_{l}
\end{aligned}
$$

The BFT impulse from the cross signal is shifted to $\pi\left(2 K_{m}-\tau_{l} / T_{s}\right)$, which is far from $\pi K_{m}$ with $\tau_{l} \ll T_{s}$. Its amplitude is low and its width is spread due to small $\tau_{l}$. Therefore, the inter-symbol interference can be negligible with small cross delay $\tau_{l}$ in general.

Shown in the upper part of Fig. 2 is the time-frequency illustration of different delayed chirp-rate signals in a symbol duration $T_{s}$. The lower part of the figure is their projections in the BFT domain. The blue impulse comes from the synchronized path component, and the red pulses correspond with the other multipath components of the same symbol. The black pulses are the BFT of the multipath components of the pervious chirp-rate symbols. In the illustration, the red and black pulses may be the interference in BFT domain to other chirp-rate components in the same symbol duration, including intra-symbol mutual interference and inter-symbol interference, respectively. Therefore, the orthogonality in the BFT domain among the chirp-rate signals is affected in a practical multipath environment.

Despite having dispersion, the multipath chirp in BFT domain are found to concentrate when $T_{s} \gg \tau_{l}$ in Eq. (27). Noting that the conclusion in the above section that $T_{s} \ll 1 / f_{d}$ is required, $T_{s}$ must be selected as a compromise for low BFT dispersion when Doppler shift and multipath delays coexist. Here, we measure the BFT dispersion performance by $d$, which is defined as

$$
d=\frac{\sum_{k \neq i} E_{k}}{E_{i}}
$$

where $E_{i}$ is the energy of the desired component in the BFT domain, and $E_{k}$ is the energy of dispersed component. Figure 3 illustrates the performances of time and Doppler dispersions. In this example, the timebandwidth product is set to a constant 10, the symbol duration $T_{s}$ varies from 2 to $100 \mu s$, and the corresponding

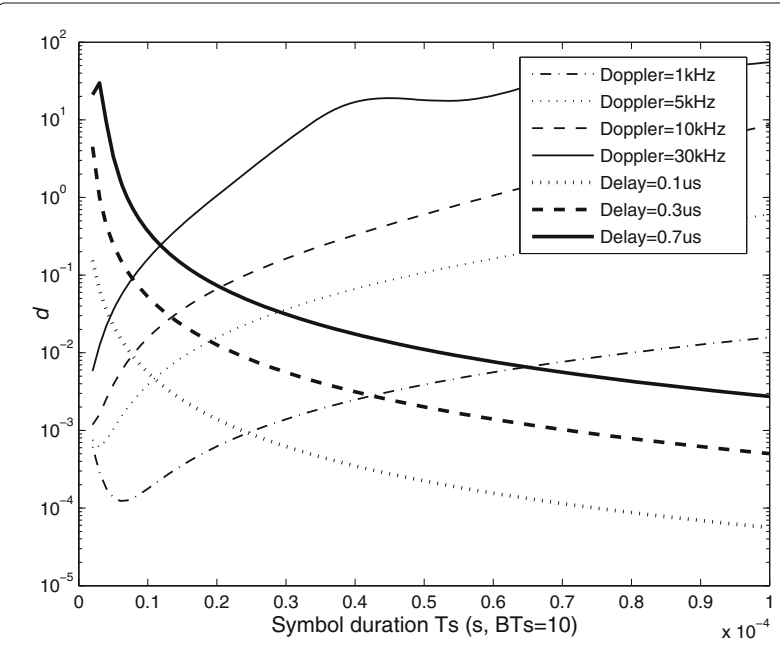

Fig. 3 Dispersion in the BFT domain due to Doppler and path delay. The figure shows the BFT dispersions in Doppler and multipath environment with different signal parameters 
bandwidth varies from 5 to $0.1 \mathrm{MHz}$. Four Doppler shifts $\{1 \mathrm{kHz}, 5 \mathrm{kHz}, 10 \mathrm{kHz}, 30 \mathrm{kHz}\}$ and three path-delays $\{0.1 \mu s, 0.3 \mu s, 0.5 \mu s\}$ are measured to obtain the BFT dispersion performance. The crossing points present the compromises of the modulation parameters under specified double dispersive channel. On the other hand, increasing $T_{s}$, i.e., decreasing $B_{m}$, in concentrating time dispersion in the BFT domain will reduce the symbol rate. Multicarrier modulation could reconcile the original data rate and dispersion requirement of BFT detection.

\subsection{Multipath and multichirp-rate signal model in the BFT domain}

Through the above analysis, the multipath signal with a single chirp rate in the $n$th symbol period is obtained in the BFT domain as

$$
\begin{aligned}
& R_{n, m}(\beta)=\operatorname{BFT}\left[r_{n, m}(t)\right] \approx \\
& b_{m}[n]\left[\alpha_{0} \delta\left(\beta-\pi K_{m}\right)+\sum_{l=1}^{L^{\prime}-1} \alpha_{l}^{\prime} \delta\left(\beta-\pi K_{m}+\Delta \tau_{l, m}^{\prime}\right)\right] \\
& +\sum_{l=L^{\prime}-1}^{L-1} b_{m}\left[n-n_{l}\right] \alpha_{l}^{\prime} \delta\left(\beta-\pi K_{m}-\Delta \tau_{l, m}^{\prime}\right)
\end{aligned}
$$

where $n_{l}=\left\lfloor\tau_{l} / T_{s}\right\rfloor,\left(\tau_{l}>T_{s}\right), \Delta \tau_{l, m}^{\prime}=\pi K_{m} \tau_{l} / T_{s}$ and $L^{\prime}$ is the number of multipaths whose delay does not exceed a symbol duration. The function $\delta(\beta)$ is an abbreviation of $\mathrm{Sa}\left[\left(T_{s}^{2}-\tau_{l}^{2}\right) \beta / 2\right] e^{\Phi(\beta)}$.

$$
\begin{aligned}
r_{n}(\beta= & \left.\pi K_{m}\right)=b_{m}[n] \alpha_{0}+\sum_{i=m+1}^{M-1} b_{i}(n) \alpha_{m, i}^{\prime} \\
& +\sum_{i=0}^{M-1} \sum_{j=1}^{N_{l}} b_{i}(n-j) \alpha_{m, i, j}^{\prime} \\
= & {\left[0, \cdots, \alpha_{0}, \alpha_{m, m+1}^{\prime}, \cdots, \alpha_{m, M-1}^{\prime}\right] \mathbf{b}(n) } \\
& +\sum_{j=1}^{N_{l}}\left[\alpha_{m, 0, j}^{\prime}, \cdots, \alpha_{m, m, j}, \cdots, \alpha_{m, M-1, j}^{\prime}\right] \mathbf{b}(n-j)+v(n)
\end{aligned}
$$

Thus, the BFT of the received multichirp-rate signal in the multipath channel is $R_{n}(\beta)=\sum_{m=0}^{M-1} R_{n, m}(\beta)+$ $v(\beta)$, where $v(\beta)$ is the Gaussian noise due to BFT being linear transform and chirp rate $K_{m}$ is assigned according to Theorem 1. Define $M$-ary source vector $\mathbf{b}(n)=$ $\left[b_{0}(n), \cdots, b_{m}(n), \cdots, b_{M-1}(n)\right]^{T}$. By the normalized D-BFT impulse of the chirp-rate signal, the D-BFT output on received multichirp-rate signal is expressed as Eq. (31). In Eq. (31), $\alpha_{m, i}^{\prime}$ denotes the mutual interference to the $m$ th chirp rate from other $i$ th chirp-rate component in a same symbol period. From the above analysis, the chirp-rate $K_{m}$ is just jammed by the chirp-rate $K_{i \geq m+1}$ components. $\alpha_{m, i, j}^{\prime}$ is the inter-symbol interference from the $i$ th chirp-rate component before $j$ symbols, and $\mathbf{h}_{m}=\left[\alpha_{0}, \alpha_{m, m, 1}, \alpha_{m, m, 2}, \cdots, \alpha_{m, m, N_{l}}\right]$ is the jamming vector from the same chirp-rate components due to multipath propagation.

Define the output vector by BFT of the received multipath signal as $\mathbf{r}(n)=\left[r_{n}\left(\pi K_{0}\right), r_{n}\left(\pi K_{1}\right), \cdots, r_{n}\right.$ $\left.\left(\pi K_{M-1}\right)\right]^{T}$. According to Eq. (31), we obtain the vector model by BFT as Eq. (32), where $\tilde{\mathbf{b}}(n)=[\mathbf{b}(n), \mathbf{b}(n-$ $\left.1), \cdots, \mathbf{b}\left(n-N_{L}\right)\right]^{T}$ is the $M$-ary source series.

\section{Detection of multichirp-rate signal in multipath environment}

Although there are a large number of interference terms in $\mathbf{H}$, many of them are zero or minute, that is, the matrix $\mathbf{H}$ is sparse. From analysis of Eq. (27) and Fig. 2, the multipath impulses by BFT are on the left of $\pi K_{m}$ in the $\beta$ domain. The further from $\pi K_{m}$, the weaker the impulses are. Therefore, the mutual collisions among impulses are not severe in practice.

The signal model by BFT in the multipath channel is a typical model of mutual and inter-symbol interference. Estimation of the matrix $\mathbf{H}$ is difficult. It is feasible to apply a training sequence and adaptive algorithms to suppress interference.

A minimum mean square error (MMSE) detector is commonly applied to mitigate interference. Because of the linear signal model in (32), MMSE detection is also available. Here, the $M \times\left(N_{l}+1\right)$ weight matrix $\mathbf{W}$ acts as the filter to $M$-channel detection. The optimal criterion in MMSE detection for the BFT of the $M$-chirp-rate signal is given by $\arg _{\mathbf{W}} \min E\left\{\left|\mathbf{b}(n)-\mathbf{W}^{H} \mathbf{r}(n)\right|^{2}\right\}$. MMSE

$$
\begin{aligned}
\mathbf{r}(n) & =\underbrace{\left[\begin{array}{cccccccccc}
\alpha_{0} & \alpha_{0,1}^{\prime} & \alpha_{0,2}^{\prime} & \cdots & \alpha_{0, M-1}^{\prime} & \alpha_{0,0,1} & \cdots & \alpha_{0, M-1,1}^{\prime} & \cdots & \alpha_{0, M-1, N_{l}}^{\prime} \\
0 & \alpha_{0} & \alpha_{1,2}^{\prime} & \cdots & \alpha_{1, M-1}^{\prime} & \alpha_{1,0,1}^{\prime} & \cdots & \alpha_{1, M-1,1}^{\prime} & \cdots & \alpha_{1, M-1, N_{l}}^{\prime} \\
\vdots & \vdots & \alpha_{0} & \ddots & \vdots & \vdots & \ddots & \vdots & \cdots & \vdots \\
0 & 0 & \cdots & \cdots & \alpha_{0} & \alpha_{M-1,0,1}^{\prime} & \cdots & \alpha_{M-1, M-1,1} & \cdots & \alpha_{M-1, M-1, N_{l}}
\end{array}\right]}_{M \times\left(N_{l}+1\right)}\left[\begin{array}{c}
\mathbf{b}(n) \\
\mathbf{b}(n-1) \\
\vdots \\
\mathbf{b}\left(n-N_{l}\right)
\end{array}\right]+\mathbf{v}(n) \\
& =\mathbf{H} \tilde{\mathbf{b}}(n)+\mathbf{v}(n)
\end{aligned}
$$


detection can be implemented by applying a training sequence and an adaptive LMS/RLS algorithm. The optimal solution is $\mathbf{W}_{\mathrm{opt}}=\mathbf{R}^{-1} \mathbf{r}$, where $\mathbf{R}=E\left[\mathbf{r}(n) \mathbf{r}(n)^{H}\right]$.

Disturbed not only by the previous symbol but also by other chirp-rate signals in the same symbol, linear MMSE detection may be inadequate for the job in a deep fading channel. Here, a decision-directed method and parallel interference cancellation (PIC) are introduced to construct a robust algorithm for the multichirp-rate signal in the multipath environment, which is called MMSE-DDPIC for short. The algorithm block diagram is shown in Fig. 4.

The interference cancellation for a chirp-rate signal is given by

$$
\hat{b}_{m}=\operatorname{sgn}\left\{\mathfrak{R}\left(y_{m}-\sum_{j \neq m}^{M} u_{m, j} \hat{b}_{j}\right)\right\}
$$

where $\hat{b}_{j}$ is the decision-directed result of channel $j$ and $u_{m, j}$ are the weights of the channel for cancellation. Define $\mathbf{U}_{m}=\left[u_{m, 1}, \cdots, u_{m, j}, \cdots, u_{m, M}\right]^{T}$ where $(j \neq m)$. By a decision-directed method, this $\mathbf{U}_{m}$ can be simultaneously adjusted with the above MMSE, which is given by

$$
\begin{aligned}
& \min _{\mathbf{W}, \mathbf{U}} E\left\{\left|b_{m}(n)-\mathbf{W}_{m}^{H} \mathbf{r}(n)+\mathbf{U}_{m}^{H} \hat{\mathbf{d}}_{m}(n)\right|^{2}\right\} \\
& \text { s.t. } \mathbf{W}_{m, \text { opt }}^{H} \cdot \mathbf{1}_{m}=1
\end{aligned}
$$

where $\hat{\mathbf{d}}_{m}(n)=\left[\hat{b}_{1}(n), \cdots, \hat{b}_{m-1}(n), \hat{b}_{m+1}(n), \cdots, \hat{b}_{M}(n)\right]$ are the decisions of other channels and $\mathbf{1}_{m}$ in constraint is a zeros vector except for its $m$ th element being one. In this MMSE criterion, $\mathbf{W}_{m}$ is the feed-forward filter, and $\mathbf{U}_{m}$ is the interference cancellation filter. The soft estimation $y_{m}(n)=\mathbf{W}_{m}^{H} \mathbf{r}(n)-\mathbf{U}_{m}^{H} \hat{\mathbf{d}}(n)$. The constraint $\mathbf{W}_{m, \text { opt }}^{H}$. $\mathbf{1}_{m}=1$ in the criterion regards the impulse position as the signature of the chirp rate in the BFT domain.

\section{Simulations}

In this section, we give simulation results of the discrete BFT demodulation and compare them with the theoretical results. In simulations, a multichirp-rate-modulated signal is implemented. The parameters of modulation include a bandwidth of $10 \mathrm{MHz}$ and a modulated symbol rate of 1 Msps. According to Theorem 1, applying orthogonal I-Q modulations, the number of chirp rates can be set to $M=10$, and the chirp rates are $K_{m}=B_{m} / T_{s}=2 k / T_{s}^{2}$, where $k= \pm 1, \pm 2, \cdots \pm 5$.

Under ideal conditions including synchronization and no noise, Fig. 5 illustrates a D-BFT result for a 10-chirprate-modulated signal. Consistent with Lemma 1 , the D-BFT of each chirp-rate signal is an impulse at the discrete $\beta$ domain without mutual interference. Meanwhile, the result verifies the condition for orthogonality in a multichirp-rate signal.

Figure 6 shows the comparison of the BER performance of D-BFT detection among single chirp-rate and 10-chirprate signals; Fig. 6 b, c shows the plots for the first and fourth rate signals, respectively. It must be pointed out that the $E_{b}$ in Fig. 6b, c is just composed of the energy of one chirp-rate component in a symbol period. From the results, we can see that the D-BFT BER performances on single-chirp-rate and multichirp-rate signals are consistent. It also proves the orthogonality of the components in the multichirp-rate signal in the D-BFT domain. At the same time, the identity of simulated and theoretical

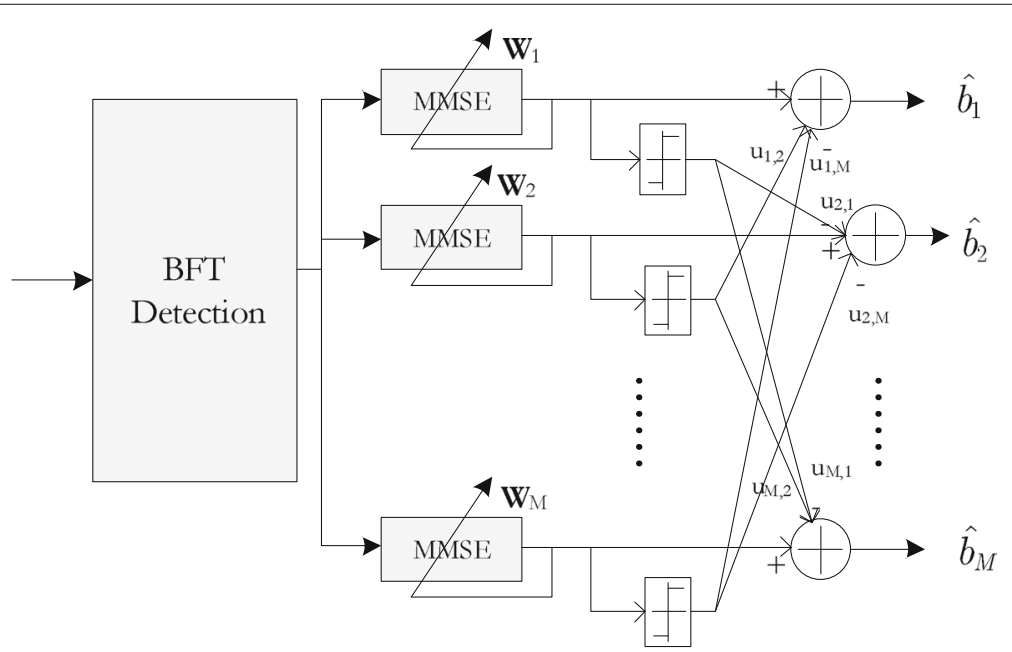

Fig. 4 Block diagram of MMSE-PIC algorithm for detecting the multichirp-rate signal in the multipath environment. The figure shows the structure of MMSE-based algorithm to suppress the interference 


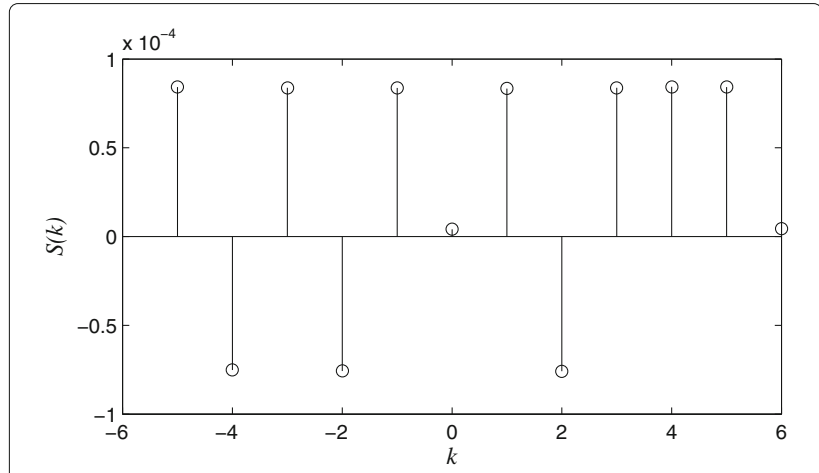

Fig. 5 D-BFT of 10-chirp-rate symbols $\{1,-1,1,-1,1,1,-1,1,1,1\}$. The figure shows the BFT output of the $M$-ary chirp-rate signal

BERs verifies the correctness of the closed-form solution of D-BFT BER in Theorem 2.

Conventional chirp-rate demodulations include matched filter (De-Chirp) and FRFT demodulations. In addition, a frequency-shift keying (FSK) signal is also a timefrequency-modulated signal. The BER performances of the three demodulation methods on time-frequency signal are given below.

- Matching filter on a chirp-rate signal (De-Chirp): $P_{e}=Q\left(\sqrt{2 E_{b} / N_{0}}\right)$.

- FRFT on a chirp binary-orthogonal keying (BOK) signal: $P_{e}=Q\left(\sqrt{E_{b} / 2 N_{0}}\right)$.

- Matching filter on FSK signal: $P_{e}=Q\left(\sqrt{E_{b} / N_{0}}\right)$.

In Fig. 7, we compare their demodulation performance with BFT detection. Simulation results show that the BER performance of BFT is better than that of FSK coherent and FRFT noncoherent detection.
Because BFT detection is a linear transformation, frequency error, or offset will cause rotation of the signal phase, we apply square-law BFT processing $\left(\mathrm{BFT}^{2}\right)$ and unipolar chirp-rate on-off keying (OOK) signal to avoid deterioration in performance. However, the performance degrades due to this square-law detection. By Eq. (21), the decline of BFT sampling amplitude caused by offset is obtained to estimate the BER of BFT ${ }^{2}$

$$
\begin{aligned}
P_{e} & =\frac{1}{2} \exp \left(-\frac{\gamma}{4}\right) \\
& =\frac{1}{2} \exp \left(\frac{-3 E_{b}}{16 N_{0}} \cdot\left|\frac{\left[\left(1-j 2 \pi f_{d} T_{s}\right) \exp \left(j 2 \pi f_{d} T_{s}\right)-1\right]}{2 \pi^{2} f_{d}^{2} T_{s}^{2}}\right|^{2}\right)
\end{aligned}
$$

where $\gamma$ is the input SNR of square-law detector.

From [25], the FRFT peak of the chirp-rate OOK signal under frequency offset is deduced as

$$
\left|S_{\alpha}(\mu)\right|_{\mu=-\cot \alpha f_{m}=\mu \csc \alpha}^{2}=\frac{A^{2} \sin ^{2}\left(\pi T_{s} f_{d}\right)}{\pi^{2} f_{d}^{2} \cdot|\sin \alpha|}
$$

where $\alpha$ is the time-frequency rotation angle in FRFT, $A$ is the amplitude of signal, and $f_{d}$ is the Doppler shift. When $|\sin \alpha| \approx T_{s} / B$, where $B$ is the bandwidth of the chirp signal and then the BER can be approximated by

$$
P_{e} \approx \frac{1}{2} \exp \left(-\frac{E_{b}}{16 N_{0}} \cdot \frac{\sin ^{2} \pi T_{s} f_{d}}{\pi^{2} f_{d}^{2} T_{s}^{2}}\right)
$$

Under different conditions, including no frequency offset, $f_{d}=100 \mathrm{kHz}$, and $f_{d}=200 \mathrm{kHz}$, BFT $^{2}$ detection performance is simulated and compared. The BER results of $\mathrm{BFT}^{2}$ in Fig. 7 are very close, which indicate that detection performance declines slightly even with large Doppler

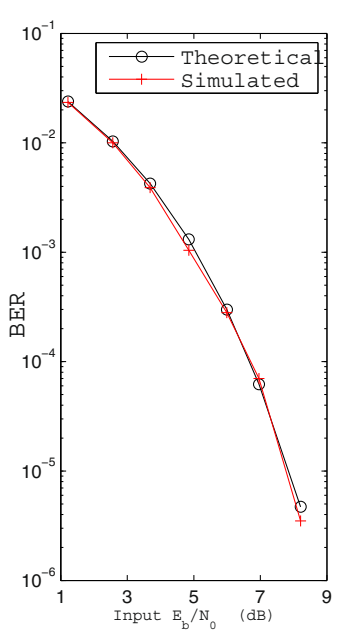

a)

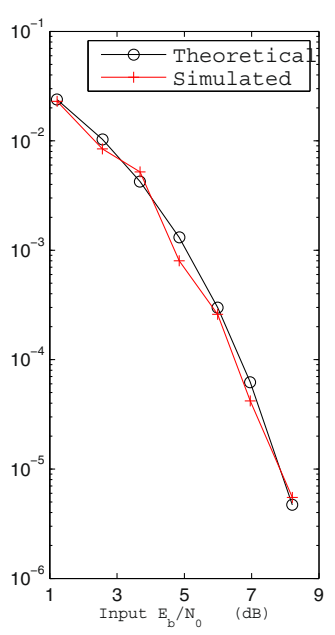

b)

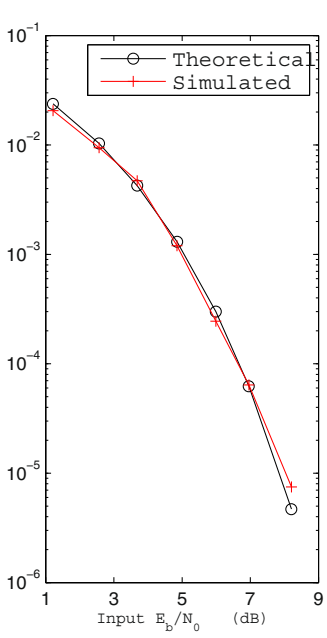

c)

Fig. 6 BER comparisons of D-BFT detection among single-chirp-rate and multichirp-rate signals. The figure compares the BER performances by the theoretical analysis and the simulation. a BFT BER of Single -Chirp-rate Signal. b 1st-rate BFT BER of the Multiple-Chirp-rate Signal. c 4th-rate BFT BER of the Multiple-Chirp-rate Signal 


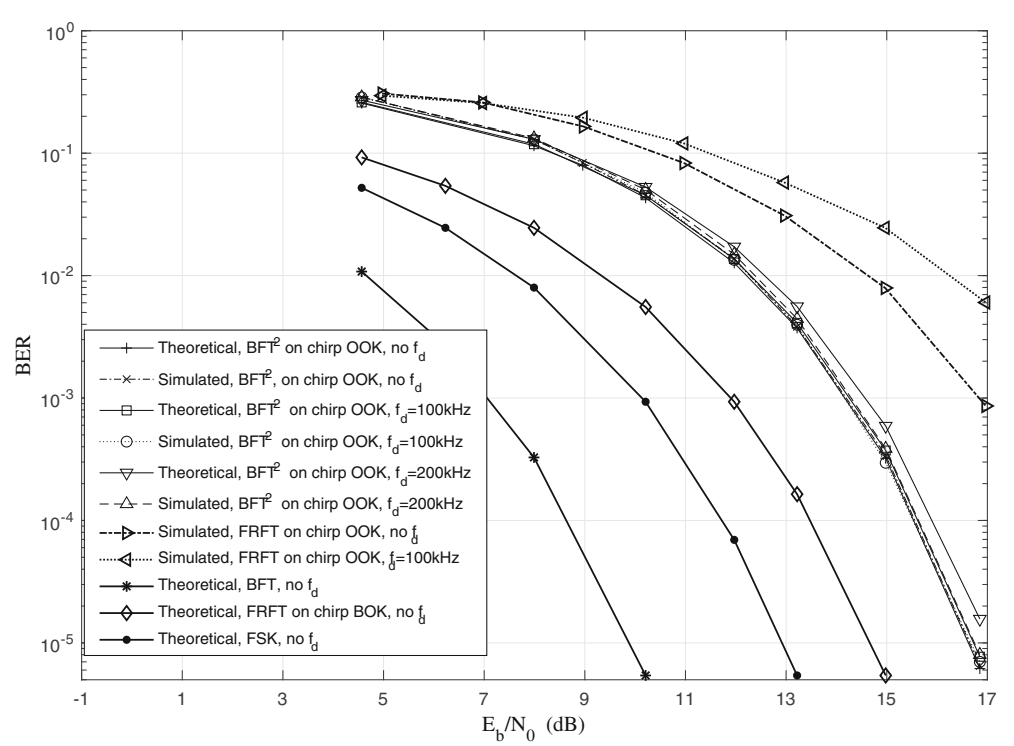

Fig. 7 Performance of D-BFT detection with frequency offset. The figure compares the BER performances among D-BFT detection, FSK in AWGN and Doppler channel

shift or frequency error. By contrast, FRFT detection exhibits a significant deterioration of BER performance at $f_{d}>100 \mathrm{kHz}$, even if it has the advantages in conventional time-frequency-selective channel compared with coherent detection $[16,25]$.

In Section 5, we analyzed the characteristics of BFT detection of the multipath chirp-rate signal. Not only does inter-symbol interference exist, but the mutual interference among different chirp-rate signals in the same symbol period also makes detection difficult. Nevertheless, due to the sparsity of the impulse interferences in the BFT domain, it is possible to mitigate them by signal processing. In the non-line-of-sight (NLOS) communications environments, the equalization and interference cancellation is a better choice for detection.

Modulated with a five-chirp-rate real signal, $10-\mathrm{Mhz}$ bandwidth, and 1-Msps symbol rate, BFT detection is simulated in the multipath environment. Two MMSEbased interference suppression algorithms are implemented according to the analysis and design presented in Section 6. The conventional LMS adaptive training process is applied to solving the optimal weight vector problem. On the other hand, we compare the BER performances at two different multipath fading environments. One is a common frequency selective fading channel, as in Fig. 8a which is the normalized channel frequency response. In Fig. 8b, we compare the BER performance of the algorithms in this channel. Auxiliary parallel interference cancellation makes the detection output close to that in flat fading. However, in this multipath environment, the performance of the MMSE-DD-PIC algorithm is only slightly better than that of MMSE detection, and for actual implementation, a choice based on computational complexity must be made. For a deep-fading frequency selective channel as shown in Fig. 9a, the BER performances are compared in Fig. 9b. The performance without interference suppressing is unacceptable. The MMSE-DD-PIC algorithm does a good job, and its performance is much better than that of simple MMSE training.
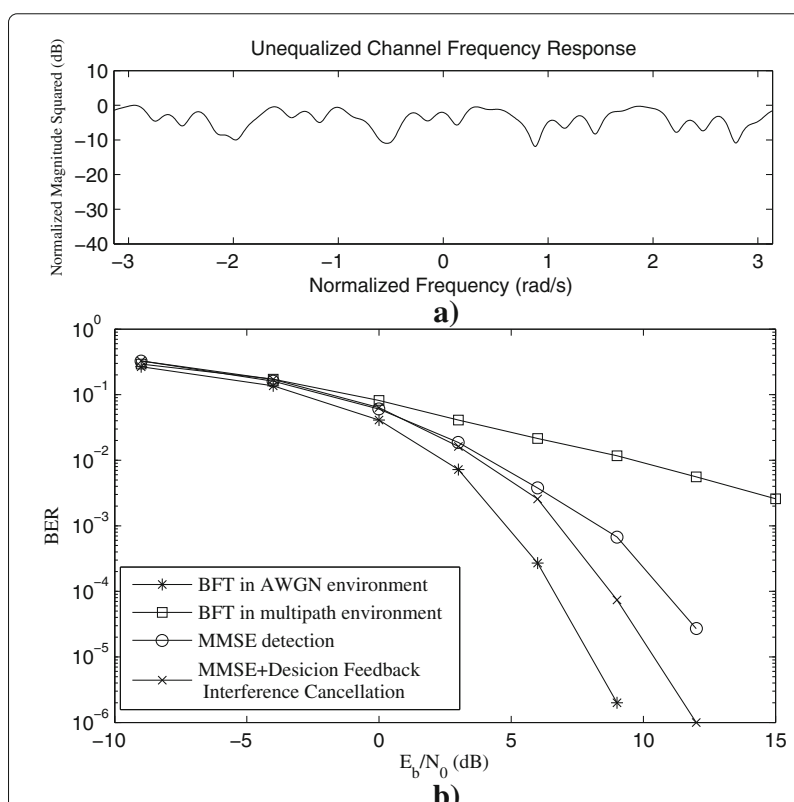

b)

Fig. 8 Performance comparisons in multipath environment. The figure shows the performance of the MMSE-based algorithm to M-ary chirp-rate signal in general frequency-selective fading environment. a Normalized frequency response of multipath fading channel. b D-BFT detection and MMSE-based algorithms 


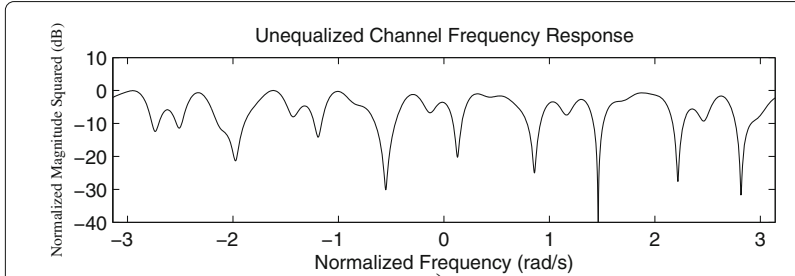

a)

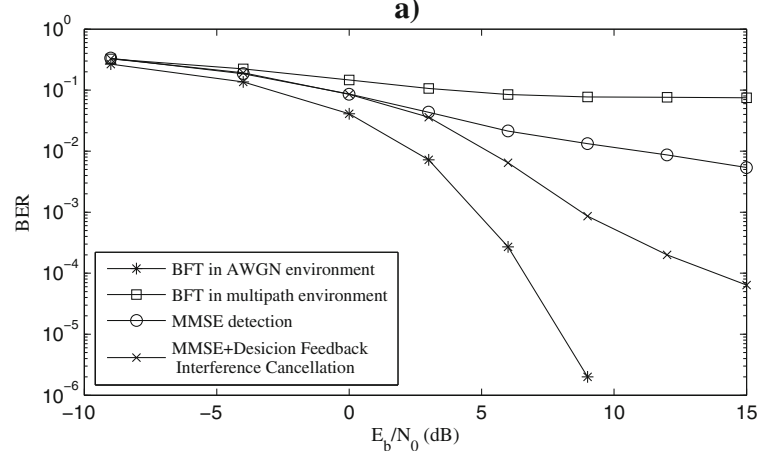

b)

Fig. 9 Performance comparisons in deep fading environment. The figure shows the performance of the MMSE-based algorithm to M-ary chirp-rate signal in deep frequency-selective fading environment. a Normalized frequency response of deep fading channel. b D-BFT detection and MMSE-based algorithms

\section{Conclusions}

The biorthogonal Fourier transform has been introduced to demodulate a multichirp-rate signal. In this paper, the detection performance of the D-BFT and BFT, including the multi-components resolution and closed-form BER, are derived. Further analyses are made in the frequencyoffset and multipath environments. The small shift of compressed $\beta$-domain impulses in the high frequencyoffset channel is a remarkable and welcome output of our research. Unfortunately, the BFT of a multichirp-rate signal is proven to have intra-symbol and inter-symbol interferences with multipath propagation or synchronization error. The theoretical result shows that the chirp modulation parameter can be adjusted to obtain the trade-off between the time and frequency dispersion. A multipath model of BFT output is constructed, and the MMSE-based algorithm is given to suppress the interference. Aided by the decision-directed PIC, the proposed detection performs well in the deep fading environment.

\section{Abbreviation}

AWGN: Additive white Gaussian noise; BER: Bit-error-rate; BFT: Biorthogonal Fourier transform; BFT ${ }^{2}$ : Square-law BFT; CSS: Chirp spread spectrum; D-BFT: Discrete BFT; FBMC: Filter bank multicarrier; FRFT: Fractional Fourier transform; ICI: Inter-carrier interference; ISI: Inter-symbol interference; LFM: Linear frequency modulation; MCP: Multi-choice precoding; MMSE: Minimum mean square error; MST: Matched signal transform; OFDM: Orthogonal frequency-division multiplexing; PIC: Parallel interference cancellation; SNR: Signal-to-noise-ratio; STDFT: Short-term discrete Fourier transform; T-F: Time-frequency

\section{Acknowledgements}

The authors would like to thank the reviewers for their thorough reviews and helpful suggestions.

\section{Funding}

This work is supported in part by the National Natural Science Foundation of China (nos. 61371107 and 61571143), the Foundation of Guangxi Broadband Wireless Communication \& Signal Processing Key Laboratory (no. GXKL061501), and the Foundation of Science and Technology on Communication Networks Laboratory (no. KX172600033).

\section{Availability of data and materials Not applicable.}

\section{Authors' contributions}

LZ is the main writer of this paper. He proposed the main idea, deduced the performance of BFT detection, completed the simulation, and analyzed the result. CYang introduced the MMSE-based algorithm in dispersive channel. CYan simulated the detection in the Doppler channel. HQ gave some important suggestions for BFT detection. All authors read and approved the final manuscript.

\section{Competing interests}

The authors declare that they have no competing interests.

\section{Publisher's Note}

Springer Nature remains neutral with regard to jurisdictional claims in published maps and institutional affiliations.

Received: 13 July 2017 Accepted: 4 January 2018

Published online: 29 January 2018

\section{References}

1. A Springer, W Gugler, et al, Spread spectrum communications using chirp signals. EUROCOMM. 2000, 166-170 (2000)

2. IEEE Standard for Local and metropolitan area networks - Part 15.4: Low-Rate Wireless Personal Area Networks (LR-WPANs). LAN/MAN Standards Committee of the IEEE Computer Society, September 2011

3. R Tao, Y Li, Y Wang, Short-time fractional Fourier transform and its applications. IEEE Trans. Signal Process. 58(5), 2568-2580 (2010)

4. J Yu, L Zhang, K Liu, Coherently distributed wideband LFM source localization. IEEE Signal Process. Lett. 22(4), 504-508 (2015)

5. BJ Wysocki, TA Wysocki, HJ Zepernick, Walsh-chirp sequences for wireless applications. J.Telecommun. Inf. Technol. 2(3), 24-28 (2001)

6. H Liu, Multicode ultra-wideband scheme using chirp waveforms. IEEE J. Sel. Areas Commun. 24(4), 885-891 (2006)

7. I Dotlić, R Kohno, Low complexity chirp pulsed ultra-wideband system with near-optimum multipath performance. IEEE Trans. Wirel. Commun. 10(1), 208-218 (2011)

8. H Shen, S Machineni, C Gupta, A Papandreou-Suppappola, Time-varying multichirp rate modulation for multiple access systems. IEEE Signal Process. Lett. 11(5), 497-500 (2004)

9. L John, I Zbigniew, H Rainer, Nanotron MDMA EBM Proposal Presentation (2005). Available: http://www.ieee802.org/22/Meeting_documents/ 2005_Nov/22-05-0102-01-0000_Nanotron_MDMA_EBM_Proposal_ Presentation.ppt.. Accessed 16 Jan 2018

10. G Matz, H Bolcskei, F Hlawatsch, Time-frequency foundations of communications. IEEE Signal Proc. Mag. 30(6), 87-96 (2013)

11. R Nissel, S Schwarz, M Rupp, Filter bank multicarrier modulation schemes for future mobile communications. IEEE J. Sel. Areas Commun (2017). preprint

12. JC Wood, DT Barry, Radon transformation of time-frequency distributions for analysis of multicomponent signals. IEEE Trans. Signal Process. 42(11), 3166-3177 (1994)

13. M-S Wang, AK Chan, CK Chui, Linear frequency-modulated signal detection using Radon-ambiguity transform. IEEE Trans. Signal Process. 46(3), 571-586 (1998)

14. S Mann, S Haykin, The chirplet transform: physical considerations. IEEE Trans. Signal Process. 43(11), 2745-2761 (1995)

15. J Zhong, Y Huang, Time-frequency representation based on an adaptive short-time Fourier transform. IEEE Trans.Signal Process. 58(10), 5118-5128 (2010)

16. M Martone, A multicarrier system based on the fractional Fourier transform for time-frequency-selective channels. IEEE Trans. Commun. 49(6), 1011-1020 (2001) 
17. J Zheng, Z Wang, ICl Analysis for FRFT-OFDM systems to frequency offset in time-frequency selective fading channels. IEEE Commun. Lett. 14(10), 888-890 (2010)

18. S Kumari, SK Rai, A Kumar, HD Joshi, AK Singh, R Saxena, Exact BER analysis of FRFT-OFDM system over frequency selective Rayleigh fading channel with CFO. Electron. Lett. 49(20), 1299-1301 (2013)

19. MR Mousavi, A Shahzadi, Hyperbolic FRFT-OFDM system BER analysis over high Doppler Rician fading channels. Electron. Lett. 51(24), 2003-2005 (2015)

20. TErseghe, N Laurenti, V Cellini, A multicarrier architecture based upon the affine Fourier transform. IEEE Trans. Commun. 53(5), 853-862 (2005)

21. D Stojanovic, I Djurovic, BR Vojcic, Multicarrier communications based on the affine fourier transform in doubly-dispersive channels. Eurasip J. Wirel. Commun. Netw. 2010(1), 868314 (2010)

22. Z Wang, L Mei, X Wang, X Sha, N Zhang, On the performance of hybrid carrier system with spectrum precoding based on WFRFT. EURASIP J. Wirel. Commun. Netw. 2017(1), 102 (2017)

23. AT Catherall, DP Williams, High resolution spectrograms using a component optimized short-term fractional Fourier transform. Signal Process. 90(5), 1591-1596 (2010)

24. F Liu, H Xu, R Tao, Y Wang, Research on resolution between multi-component LFM signals in the fractional Fourier domain. Sci. China Inf. Sci. 55(6), 1301-1312 (2012)

25. B Deng, R Tao, E Chen, in 2006 8th international Conference on Signal Processing. A novel method for binary chirp-rate modulation and demodulation, (2006), pp. 1-4. https://doi.org/10.1109/ICOSP.2006.345831

26. E Sejdic, I Djurovic, L Stankovic, Fractional Fourier transform as a signa processing tool: an overview of recent developments. Signal Process. 91(6), 1351-1369 (2011)

27. A Papandreou-Suppappola, RL Murray, BG Lem, GF Boudreaux-Bartels, Group delay shift covariant quadratic time-frequency representations. IEEE Trans. Signal Process. 49(11), 2549-2564 (2001)

28. H Shen, A Papandreou-Suppappola, Wideband time-varying interference suppression using matched signal transforms. IEEE Trans.Signal Process. 53(7), 2607-2612 (2005)

29. B Wang, X Li, Analysis algorithm to frequency rate of LFM signal based on biorthogonal Fourier transform (in Chinese). J. Electron. Inf. Technol. 31(7), 1620-1623 (2009)

30. H Wang, Y Li, B Wang, High-order bi-orthogonal Fourier transform and its applications in non-stability signal analysis. IEICE Trans.Inf. Syst. E98-D(1), 189-192 (2015)

31. Q Lin, R Tao, S Zhou, et al, Detection and parameter estimation of multicomponent LFM signal based on the fractional Fourier transform. Sci. China Inf. Sci. 47(2), 184-198 (2004)

32. B Porat, Digital processing of random signals. (Prentice Hall, Englewood Cliffs, 1994)

\section{Submit your manuscript to a SpringerOpen ${ }^{\circ}$ journal and benefit from:}

- Convenient online submission

- Rigorous peer review

- Open access: articles freely available online

- High visibility within the field

- Retaining the copyright to your article

Submit your next manuscript at $\gg$ springeropen.com 